\title{
Hypertension in pregnancy
}

\author{
Motha MBC Jayasundara C
}

\section{INTRODUCTION}

Hypertension, defined as a systolic blood (SBP) $\geq 140 \mathrm{~mm} \mathrm{Hg}$ and /or diastolic blood pressure (DBP) $\geq 90 \mathrm{mmHg}$ is the commonest medical disorder encountered in pregnancy. It includes women with chronic hypertension (hypertension prior to 20 weeks of pregnancy or present at the booking visit including pre-existing hypertension), gestational hypertension ( hypertension presenting after 20 weeks without proteinuria) and pre-eclampsia ( hypertension presenting after 20 weeks with significant proteinuria).

\section{MEASUREMENT OF BLOOD PRESSURE}

Blood pressure should be measured with the woman seated and feet supported. Measurements should be taken after two to three minutes resting in this position. A standard size cuff should be used for women with an arm circumference of less than $33 \mathrm{~cm}$ and a large cuff used for arm circumference above $33 \mathrm{~cm}$. The bladder of the sphygmomanometer should encircle 2/3rds of the upper arm circumference. The cuff should be inflated above $20-30 \mathrm{mmHg}$ of the palpable systolic blood pressure and deflated at a rate of $2 \mathrm{mmHg}$ per second, recording $\mathrm{BP}$ to the nearest $2 \mathrm{mmHg}$. Korotkoff phase I and $\mathrm{V}$ should be considered as systolic and diastolic blood pressure respectively.

\section{PREEXISTING HYPERTENSION}

In addition to women who are known to be hypertensive, women detected with hypertension prior to 20 weeks, especially those with high blood pressure in the first trimester should be suspected of having preexisting hypertension. Preconception care

Correspondence: Dr. Motha MBC, Jayasundara C E mail-

Competing interests: None is vital in women with preexisting hypertension in order to achieve normotension prior to conception, modification of antihypertensive medication and screening for target organ damage. Angiotensin converting enzyme inhibitors, angiotensin receptor blockers and thiazide diuretics are teratogenic and should preferably be modified at the pre conception stage. Women with a secondary cause for hypertension (Eg: pheochromocytoma, renal artery stenosis etc.) should be referred to the relevant specialist for advise on management of hypertension during pregnancy. ${ }^{1} \quad$ Aspirin $75 \mathrm{mg}$ daily should be commenced at 12 weeks and continued until the birth of the baby. ${ }^{1}$

\section{GESTATIONAL HYPERTENSION}

Hypertension occurring in the second half of pregnancy in a previously normotensive woman, without significant proteinuria or other features of pre-eclampsia, is termed gestational or pregnancy induced hypertension. It complicates $6-7 \%$ of pregnancies and resolves post partum. ${ }^{2}$ In gestational hypertension, blood pressure usually normalises by six weeks post partum. Women with gestational hypertension should be referred to a secondary care setting for management of pregnancy.

\section{PREECLAMPSIA}

Pre-eclampsia usually occurs after 20 weeks gestation and is characterised by significant proteinuria and hypertension. Pre-eclampsia has a complex pathophysiology, the primary

cause being abnormal placentation. During normal pregnancy, the villous cytotrophoblast

invades the inner third of the myometrium, and spiral arteries lose their endothelium and most of their muscle fibers. These structural modifications are associated with functional alterations, such that spiral arteries become low resistance vessels, and thus less sensitive to vasoconstrictivesubstances. Abnormal placentation due to suboptimal invasion on the spiral arteries leads to increased uterine arterial resistance with higher sensitivity to vasoconstrictors and thus chronic placental ischemia and oxidative stress. Oxidative stress induces release of substances such as free radicals, oxidized lipids, cytokines, and serum soluble vascular endothelial growth factor into the systemic circulation. These abnormalities are responsible for endothelial dysfunction. ${ }^{3}$ Perfusion is decreased to virtually all organs, which is secondary to intense vasospasm due to an increased sensitivity of the vasculature to pressor agents. Perfusion is further compromised by activation of the coagulation cascade, especially platelets, with attendant microthrombi formation. Additionally, plasma volume is decreased by loss of fluid from the intravascular space, further compromising organ blood flow.

An automated reagent strip reading device could be used to detect proteinuria and a result of $1+$ or more should prompt quantification of proteinuria using a spot protein: creatinine ratio or a 24 hour urine collection. Presence of significant proteinuria is diagnosed when the urinary protein: creatinine ratio is greater than $30 \mathrm{mg} / \mathrm{mmol}$ or 24 hour urine collection shows greater than $300 \mathrm{mg}$ of protein.

Overall pre-eclampsia complicates $5-6 \%$ of pregnancies, but this figure increases to up to $25 \%$ in women with pre-existing hypertension. ${ }^{4}$ When gestational hypertension is diagnosed after 36 weeks of pregnancy, the risk falls to $10 \%$. An estimated 50000 women die annually from pre-eclampsia worldwide due to placental abruption, intra-abdominal haemorrhage, cardiac failure, and 
multi-organ failure. ${ }^{5}$ Women with hypertension should be advised to seek immediate care if they develop symptoms suggestive of preeclampsia which include severe headache, vision disturbances such as blurring or flashing before the eyes, right hypochondrial or epigastric pain, vomiting or sudden swelling of the feet or face.

In the presence of any of the above features and significant proteinuria the woman should be admitted and blood pressure closely monitored. The blood pressure should be monitored four times a day. The full blood count, renal functions including electrolytes and hepatic transaminases should be monitored twice weekly in those with mild hypertension and thrice weekly in those with moderate hypertension and beyond.

Severe preeclampsia is diagnosed in the presence of severe hypertension and proteinuria or mild or moderate hypertension with one of severe headache, blurring of vision or flashing of lights, right hypochondrial pain, vomiting, papilloedema, sustained clonus, right hypochondrial tenderness, HELLP (haemolysis, elevated liver enzymes, low platelets) syndrome, platelet count $<100 \times 10^{9}$ / litre and AST or ALT $>70 \mathrm{IU} / \mathrm{L}$.

In women with severe preeclapmsia, maintenance fluids should be limited to $80 \mathrm{ml} /$ hour.

\section{ECLAMPSIA}

An eclamptic seizure may be preceded by severe preeclampsia or mild hypertension without proteinuria. An eclamptic seizure usually lasts 60-90 seconds. A postictal phase may be present with confusion and agitation. The timing of an eclamptic seizure can be antepartum (53 percent), intrapartum (19 percent), or postpartum (28 percent). ${ }^{6}$

Initial management of an eclamptic seizure includes protecting the airway and minimizing the risk of aspiration by placing the woman on her left side, suctioning her mouth, and administering oxygen. Magnesium sulphate is the drug of choice in preventing further seizures.
Magnesium sulphate should also be considered in women with severe preeclampsia in whom birth is planned within 24 hours. A loading dose of $4 \mathrm{~g}$ of magnesium sulphate should be administered intravenously over 5 minutes, followed by an infusion of $1 \mathrm{~g}$ per hour maintained for 24 hours. Recurrent seizures are managed with a repeat dose of 2-4 g given over 5 minutes. $^{7}$

\section{WHEN TO START ANTIHYPERTENSIVES}

Those with mild (systolic blood pressure 140-149 $\mathrm{mmHg}$ and diastolic 90-99 $\mathrm{mmHg}$ ) hypertension could be managed as outpatients with advise on weekly measurement of blood pressure. Those with moderate hypertension (systolic blood pressure 150-159 $\mathrm{mmHg}$ and diastolic 100$109 \mathrm{mmHg}$ ) should be commenced on medication with one of oral labetolol, methyldopa or nifedipine (slow release) tablets. The mortality and morbidity of women with severe hypertension (> 160/110 mm Hg), usually secondary to severe preeclampsia, remain considerable. Management of severe hypertension involves adequate blood pressure control, often using parenteral agents. Parenteral hydralazine or labetalol are considered first line agents. A Cochrane review showed no evidence that one parenteral agent had superior effectiveness. ${ }^{8}$ Available data also favour the use of oral nifedipine in the management of severe hypertension in pregnancy. ${ }^{9}$

Because of contraction of circulating plasma volume, women may be very sensitive to relatively small doses of antihypertensive agents, risking abrupt reductions in blood pressure. Good control of hypertension in severe pre-eclampsia does not halt the progression of the disease, but reduces the incidence of complications such as cerebral haemorrhage.

\section{MEDICATIONS USED IN HYPERTENSION OF PREGNANCY}

The long term safety for the fetus with use of methyldopa has been well demonstrated, but recommendations suggest avoiding the use of methyldopa in the postpartum period because it can cause depression in some women. However, it is only a mild antihypertensive agent and has a slow onset of action (three to six hours), and therefore may be less effective for severe hypertension. ${ }^{10}$ Methyldopa is started at a dose of $250 \mathrm{mg}$ or $500 \mathrm{mg}$ bd and increased upto a maximum of $2 \mathrm{~g}$ per 24 hours. The drug may result in an elevation of liver transaminases (in up to $5 \%$ of women) or a positive Coomb's test (although haemolytic anaemia is uncommon).

Oral labetolol should be commenced at a dose of $100 \mathrm{mg}$ twice daily and increased upto $1200 \mathrm{mg}$ of a total daily dose. Slow release nifedipine should be commenced at a dose of $20 \mathrm{mg}$ daily and increased up to $120 \mathrm{mg}$ daily. Hydralazine should be commenced at a dose of $5 \mathrm{mg}$ IV and repeated every 30 minutes to a maximum of $20 \mathrm{mg}$ IV (or $30 \mathrm{mg}$ IM). It could also be given as an infusion at a rate of 50 $150 \mu \mathrm{g} /$ minute. Hydralazine should be given after a colloid challenge to reduce the reflex tachycardia, and abrupt hypotension, precipitated by vasodilatation of a volume contracted circulation.

\section{TARGET BLOOD PRESSURE}

Overzealous blood pressure control may lead to placental hypoperfusion, as placental blood flow is not autoregulated, which in turn will compromise the fetus. Once treatment is started, target blood pressure is also controversial, but many practitioners advocate a mean arterial pressure of $125 \mathrm{mmHg}$. Eg: a blood pressure 150/100 mm Hg.

In women with chronic hypertension and absence of target organ damage, the aim is to maintain BP below 150/100 mmHg during pregnancy, while in the presence of target organ damage secondary to chronic hypertension, (Eg: renal impairment, left ventricular hypertrophy, hypertensive retinopathy) the blood pressure should be maintained below $140 / 90 \mathrm{mmHg}$. There is no evidence that pharmacological treatment of chronic or gestational hypertension protects against the development of pre-eclampsia. Changes in diet or bed rest have not been shown to provide maternal or fetal benefit. 
The blood pressure should be monitored weekly in mild hypertension, twice weekly in moderate hypertension and four times a day in severe hypertension. In women with mild hypertension presenting before 32 weeks, or at high risk of pre-eclampsia, blood pressure and urine for significant proteinuria should be assessed twice weekly.

\section{ANTENATAL FETAL MONITORING}

Hypertension in pregnancy and especially preeclampsia is associated with increase fetal morbidity and mortality. These include oligohydroamnios, fetal growth restriction (FGR), absent or reversed end diastolicflow in theumbilicalartery by doppler velocimetry, placental abruption and even fetal demise. So it's vital that fetal morbidity is detected early in hypertensive disease of pregnancy. ${ }^{11}$ As much of fetal morbidity and mortality in chronic hypertension is due to associated superimposed preeclampsia or fetal growth restriction, the management should be focused on detecting these early. ${ }^{12}$ Fetal growth, amniotic fluid volume assessment and umbilical artery doppler should be assessed at 28-30 weeks and 32-34 weeks. If there is no abnormality at these scans further testing can be deferred unless new problem arises . If there is a clinical indication an earlier ultrasound assessment can be done. Antenatal non stress test (CTG) is indicated only if there are abnormal fetal movements. ${ }^{i}$

In pregnancy induced hypertension (PIH) and mild preeclampsia, a growth scan, amniotic fluid assessment and an umbilical artery Doppler ( UAD) is indicated if diagnosed prior to 34 weeks but its value is controversial if its diagnosed after 34 weeks and if the fetal growth was normal prior to 34 weeks. $^{1}$ If FGR is suspected in a preeclamptic women, the fetal growth assessment should be performed serially every three weekly to detect worsening FGR.${ }^{13}$ If FGR is worsening more frequent amniotic fluid assessment and Doppler studies of umbilical arteries are needed. A CTG is beneficial if there is reduced fetal movements, unexpected antepartum hemorrhage, abdominal pain and if there is a deterioration in maternal condition. ${ }^{1}$

If decided to deliver prior to 34 weeks, a course of antenatal corticosteroids for fetal lung maturation is recommended as it's proven to reduce the incidence of respiratory distress syndrome and intraventricular haemorrhages in preterm neonates. ${ }^{14}$

\section{PLANNING DELIVERY}

Delivery is the only cure for hypertension in pregnancy. In a woman with preeclampsia, delivery should be planned when the woman reaches 36-37 weeks of gestation, irrespective of the degree of preeclampsia. Expectant management is also not justified if preeclampsia occurs prior to 14 weeks in view of high risk of maternal complications and poor fetal prognosis. At $34-37$ weeks, management depends on the severity of pre-eclampsia. Expectant management is possible for mild preeclampsia to limit the risk of induced preterm delivery, but for severe preeclampsia, delivery remains the rule due to the increased risk of maternal and fetal complications. For patients with severe preeclampsia between 24 and 34 weeks of gestation, data are insufficient to recommend "interventionist" versus expectant management. ${ }^{15}$

\section{INTRAPARTUM CARE}

Antihypertensives should be continued in labour. During labour blood pressure should be measured hourly in women with mild and moderate hypertension and continuously in those with severe hypertension. In women with mild, moderate and severe hypertension in whom blood pressure is controlled the second stage of labour need not be prolonged. Operative delivery should be considered in women who remain to have severe hypertension in the second stage of labour that is poorly responsive to antihypertensives.

Fetal monitoring in labour does not differ much from monitoring in any other high risk pregnancy, and if available continuous fetal monitoring should be considered especially if there is associated FGR. ${ }^{1}$ Fetal indication for expeditious delivery include repetitive late decelerations, severe variable decelerations and short term variability less than $3 \mathrm{bpm} .{ }^{12}$

\section{POST NATAL MANAGEMENT}

Blood pressure should be measured daily in the first two days after birth, and at least once between day 3 and 5 . In women with preeclampsia and on treatment during the antenatal period, blood pressure should be monitored 4 times a day while an inpatient. Antihypertensive treatment may be reduced when the blood pressure falls below 130/80 mmHg. Women who have not been on antihypertensives during the antenatal period and whose blood pressure is $150 / 100 \mathrm{mmHg}$ or above should be commenced on antihypertensives.

In women who are breastfeeding, labetolol, nifedipine, captopril, enalapril, atenolol and metoprolol can be used safely.

In women with preeclampsia, serum creatinine, platelet count and serum transaminases should be performed after 48-72 hours of birth and repeated if results are abnormal. Proteinuria should be assessed at 6 weeks in women with preeclampsia and if elevated should be reviewed with repeat assessment at 3 months. If proteinuria persists referral to a renal specialist should be sought.

On discharge, advise should be given with regard to frequency of blood pressure assessment, thresholds for stopping or starting treatment and when to attend for a review. In women who had preeclampsia during the antenatal period and were on treatment, blood pressure should be monitored every 1-2 days until antihypertensives are stopped. Arrangements should be made for medical review of women who remain on antihypertensive treatment 2 weeks after birth and a 6 week review for all women with gestational hypertension. Those who remain hypertensive beyond 6 weeks postpartum should be referred to a medical clinic for specialist assessment of hypertension and follow up care. 


\section{REDUCING THE RISK OF HYPERTENSIVE DISORDERS}

Low dose aspirin has been shown to reduce the relative risk of preeclampsia by $19 \% .{ }^{16}$ Women with hypertensive disease during a previous pregnancy, chronic kidney disease, systemic lupus erythematosus or antiphospholipid syndrome, type 1 or 2 diabetes mellitus and chronic hypertension should be commenced on $75 \mathrm{mg}$ of aspirin daily from 12 weeks onwards until the birth of the baby. Women with more than one moderate risk factor which includes first pregnancy, age equal or more than 40 years, pregnancy interval of more than 10 years, body mass index of more than $35 \mathrm{Kg} / \mathrm{m}^{2}$, family history of preeclampsia and multiple pregnancy should also be commenced on a similar regimen of aspirin.

\section{RISK OF RECURRENCE OF HYPERTENSIVE DISORDER IN SUBSEQUENT PREGNANCY}

Women who experience hypertension in a first pregnancy are at increased risk in a subsequent pregnancy ${ }^{17}$ Certain factors influence this risk. The earlier the onset of hypertension in the first pregnancy, the greater the risk of recurrence. ${ }^{18}$ The type of hypertensive disorder influences recurrence. One study reported a recurrence risk of $19 \%$ for gestational hypertension, $32 \%$ for pre-eclampsia, and $46 \%$ for pre-eclampsia superimposed on pre-existing chronic hypertension. ${ }^{6}$ In addition, severe isolated IUGR has been identified as a risk factor for developing hypertension in a subsequent pregnancy. ${ }^{6}$ In women with preeclampsia, the risk of preeclampsia in a future pregnancy is 1 in 6 . However, the risk rises to 1 in 4 in the presence of a history of severe preeclampsia, HELLP syndrome or eclampsia prior to 34 weeks and 1 in 2 if birth occurred before 28 weeks.

Approximately $20 \%$ of women with pre-eclampsia develop hypertension or microalbuminuria during longterm follow-up, and the risk of subsequent cardiovascular and cerebrovascular disease is doubled in women with pre-eclampsia and gestational hypertension compared with age-matched controls. ${ }^{19}$ Children born after pre-eclamptic pregnancies and who are relatively small at birth, have an increased risk of stroke, coronary heart disease, and metabolic syndrome in adult life. ${ }^{20} 2122$

\section{REFERENCES}

1. Hypertension in pregnancy NICE guideline (CG107) 2010

2. Walker JJ. Pre-eclampsia. A broad overview of the epidemiology, pathophysiology, and management of pre-eclampsia . Lancet 2000;356:12605.

3. Roberts JM. Endothelial dysfunction in preeclampsia. Semin Reprod Endocrinol. 1998;16:5-15.

4. Saudan P, Brown MA, Buddle ML, et al. Does gestational hypertension become pre-eclampsia? Br J Obstet Gynaecol 1998;105:1177-84.

5. Broughton Pipkin F. Risk factors for preeclampsia. N Engl J Med 2001;344:9256.

6. Mattar F, Sibai BM. Eclampsia. VIII. Risk factors for maternal morbidity. Am J Obstet Gynecol. 2000;182(2):307-312.

7. The Eclampsia Trial Collaborative Group Which anticonvulsant for women with eclampsia? Evidence from the Collaborative Eclampsia Trial. Lancet 1995 345:1455-63.

8. Duley L, Henderson-Smart DJ, Meher S. Drugs for treatment of very high blood pressure during pregnancy. Cochrane Database Syst Rev. 2006;(3):CD001449.

9. Shekhar S, Sharma C, Thakur S, Verma S. Oral nifedipine or intravenous labetalol for hypertensive emergency in pregnancy: a randomized controlled trial. Obstet Gynecol 2013;122:1057-63.

10. World Health Organization (WHO). Chapter 2: Improving Maternal and Perinatal Health, 4.1.5: Treatment of mild to moderate hypertension trial. In: HRP Biennial Technical Report 2009-2010. Geneva: WHO; 2011.

11. Sibai B, Dekker G, Kupferminc M. Preeclampsia. Lancet 2005;365:785-99.

12. Cohen WR, editor. Obstetric medicine: Management of medical disorders in pregnancy. 6th edition. United States: PMPH-USA; 2013.

13. Perni S, Chervenak F, Kalish R, Magherini-Rothe S, Predanic M, Streltzoff $J$, et al. Intraobserver and interobserver reproducibility of fetal biometry.
Ultrasound in Obstetrics and Gynecology 2004:24:654-8.

14. Shah DM, Shenai JP, Vaughn WK Neonatal outcome of premature infants of mothers with preeclampsia. Semin Perinatol 1994;15:264-7.

15. Churchill D, Duley L. Interventionist versus expectant care for severe preeclampsia before term. Cochrane Database Syst Rev. 2002;(3):CD003106.

16. Duley L, Henderson-Smart DJ, Knight M, King JF. Antiplatelet agents for preventing pre-eclampsia and its complication (review). Cochrane Database Syst Rev 2003;(4):CD004659

17. Hargood JL, Brown MA. Pregnancyinduced hypertension; recurrence rate in second pregnancies. Med $\mathrm{J}$ Austral 1991;154:376-87.

18. Zhang J, Troendle JF, Levine RJ. Risks of hypertensive disorders in the second pregnancy. Paediatr Perinat Epidemiol 2001;15:226-31.

19. Smith GC, Pell JP, Walsh D. Pregnancy complications and maternal risk of ischemic heart disease: a retrospective cohort study of 129,290 births. Lancet. 2001;357:2002-2006.

20. Meads CA, Cnossen JS, Meher S, et al. Methods of prediction and prevention of preeclampsia: systematic reviews of accuracy and effectiveness literature with economic modelling. Health Technol Assess. 2008;12:1-270.

21. Osmond $C$, Kajantie E, Forsén TJ, Eriksson JG, Barker DJ. Infant growth and stroke in adult life: the Helsinki Birth Cohort Study. Stroke. 2007;38:264-270.

22. Eriksson JG, Forsén T, Tuomilheto J, Osmond C, Barker DJ. Early growth and coronary heart disease in later life: longitudinal study. BMJ. 2001;322:949953. 